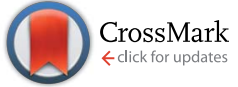

Cite this: RSC Adv., 2016, 6, 6223

Received 4th December 2015 Accepted 5th January 2016

DOI: $10.1039 / \mathrm{c} 5 \mathrm{ra} 25898 \mathrm{~g}$

www.rsc.org/advances

\section{Formation of drug nanocrystals under nanoconfinement afforded by liposomes $\uparrow$}

\author{
D. Cipolla, ${ }^{\star a b}$ H. Wu, ${ }^{b}$ S. Salentinig, ${ }^{c}$ B. Boyd, ${ }^{d}{ }^{\text {T. Rades, }}{ }^{e}$ D. Vanhecke, ${ }^{f}$ A. Petri-Fink, ${ }^{f}$ \\ B. Rothin-Rutishauser, ${ }^{f}$ S. Eastman, ${ }^{g}{ }^{T}$. Redelmeier, ${ }^{\text {h }}$ I. Gonda ${ }^{b}$ and H. K. Chan ${ }^{a}$
}

Nanocrystals of drug substances have important therapeutic applications, but their preparation is often difficult due to size control in bottom up approaches, or energetic milling and surface activation in top down processing. In this study, confinement within liposome nanocompartments is demonstrated to enable drug crystallization with a high aspect ratio but limited growth resulting in nanocrystals, using a simple freeze-thaw process which is anticipated to be amenable to large scale preparation. After the freeze-thaw, cryo-transmission electron microscopy (cryoTEM) imaging and cryo-electron tomography revealed that the majority of the liposomes contained a single drug nanocrystal, observed to physically stretch but not burst the liposomes, and the composition of the freeze-thaw medium altered the aspect ratio of the drug nanocrystals. Small angle X-ray scattering and dynamic depolarized light scattering were used to confirm the asymmetric nature of particles in suspension to exclude the cryoTEM preparation process as a contributor to the particle morphology. In assessing potential use in controlled release drug delivery, the in vitro release rate of ciprofloxacin from liposomes containing the nanocrystals revealed that the rate of dissolution of the nanocrystals became the rate controlling step, in contrast to the lipid bilayer rate controlling function prior to the formation of the crystals. The ability to modulate the release rate of the active ingredient in a complex formulation using simple physical means (e.g., freeze/thaw) is an attractive possibility, especially in highly regulated industries such as pharmaceuticals where qualitative and quantitative changes of composition would require extensive safety evaluations.

\section{Introduction}

The use of drug nanocrystals is gaining interest in many industries. In particular, it is used to modify drug dissolution and release behaviour as well as to make medicines with high drug loading and the ability to target specific tissues via the central vascular system after oral or parenteral administration. In the context of pulmonary drug delivery, there are a number of patient populations with lung infections that may optimally be treated by different antibiotic release profiles, depending upon

${ }^{a}$ Faculty of Pharmacy, The University of Sydney, Australia. E-mail: dcip8510@uni. sydney.edu.au

${ }^{b}$ Aradigm Corporation, Hayward, CA 94545, USA. E-mail: cipollad@aradigm.com

'Laboratory for Biointerfaces, Department Materials meet Life, Empa, Swiss Federal Laboratories for Materials Science and Technology, St. Gallen, Switzerland. E-mail: stefan.salentinig@gmail.com

${ }^{d}$ Monash Institute of Pharmaceutical Sciences, Monash, Australia

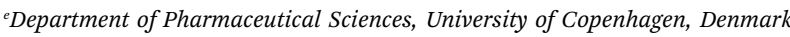

${ }^{f}$ Adolphe Merkle Institute, Université de Fribourg, Fribourg, Switzerland

${ }^{g}$ ProNAi Therapeutics, Vancouver, BC, Canada

${ }^{h}$ Northern Lipids Inc., Burnaby, Canada

$\dagger$ Electronic supplementary information (ESI) available. See DOI: $10.1039 / \mathrm{c} 5 \mathrm{ra} 25898 \mathrm{~g}$ the location of the infection (e.g., extracellular versus intracellular ${ }^{1}$ ), the presence of biofilm, and the sensitivity of the infection to the antibiotic. For example, for intracellular infections, e.g., lung macrophages harbouring nontuberculous mycobacteria (NTM), ${ }^{1}$ it may be more effective to encapsulate drug in nanocarriers such as liposomes, so that when the liposomes are taken up by the macrophages, a delayed or slow release occurs. We hypothesized that crystallization of drug under confinement inside liposomes would provide a reduced rate of drug availability by retaining the drug in a non-sink environment. In contrast, in the context of oral drug delivery, the preparation of nanocrystals is a sought after strategy for enhancing dissolution rates and bioavailability, in the absence of a liposome compartment. However, manufacturing processes that can handle incorporation of drug nanocrystals into solid oral dose forms (e.g., tablets and capsules) have similarly been a major challenge and have limited translation into more effective medicines. New methods to prepare nanocrystals have potential for wider applications beyond the pharmaceutical field.

A controlled acceleration of drug release from liposomes with minimal change in composition is achievable by the addition of surfactant to the liposomes under hyperosmotic 
conditions. ${ }^{2}$ In contrast, there is no apparent methodology to attenuate the rate of drug release by simple addition of a component to the liposomes to reduce the permeability of the lipid membrane. A slower release of drug may be achieved, without changing the composition of the initial formulation, if the drug is converted from a soluble form within the liposomes into a solid form with a slow dissolution profile, and thereby introduce a rate-limiting step prior to transport across the liposomal membrane. Doxorubicin, ${ }^{3-5}$ topotecan $^{6}$ and vinorelbine $^{7}$ formed drug crystals after being loaded into liposomes. However, these crystals were formed immediately after drug loading rather than at a later time under the control of the preparation process. It would be desirable to have methods to convert metastable supersaturated solutions of drug within liposomes in a controlled manner into nanocrystals to engineer the rates of drug transport into the biological milieu. Some drugs used in pulmonary delivery via inhalation, such as ciprofloxacin, have been prepared where the drug is present in solution in a supersaturated state in a number of liposome formulations but not in crystalline form. ${ }^{8-10}$ The ultimate hypothesis governing the experimental approach to prepare drug nanocrystals in this report is that applying a simple freezethaw step to supersaturated formulations would induce conversion of the soluble drug into drug nanocrystals with the ice crystals inside the vesicles serving as nucleation sites for drug crystallization. This provides the possibility for 'finetuning' drug delivery rates by making various mixtures of dissolved or solid drug, with liposomally encapsulated drug in the form of saturated solution or encapsulated drug nanocrystals. It is the latter which is the subject of this publication.

Therefore to test the approach to formation of nanocrystals and impact on drug release, liposomes with a mean size of 70$90 \mathrm{~nm}$ loaded with ciprofloxacin were used. ${ }^{11,12}$ To stabilize liposomes to the freeze-thaw process, disaccharides including trehalose and sucrose have been used effectively in previous studies, ${ }^{\mathbf{1 3 - 1 5}}$ and analogous to lyophilization, maximum protection to freeze-thaw is afforded when the cryoprotectant is present both internally and externally to the liposome. ${ }^{16,17}$ Thus, sucrose and trehalose were evaluated as cryoprotectants for the liposomal ciprofloxacin preparations. Following formation of nanocrystals within the liposomes after freeze-thaw, the liposome vesicle size distribution was determined by dynamic light scattering. The size and structure of these nanocrystals were characterized using cryoTEM imaging, cryo-electron tomography, polarized and depolarized dynamic light scattering (DDLS), and small angle X-ray scattering (SAXS). The drug release rate was also measured in an in vitro release assay ${ }^{18}$ to determine whether the presence of the drug nanocrystals had an effect on the release profile.

\section{Results and discussion}

\section{Formation of drug nanocrystals in liposomal ciprofloxacin using a freeze-thaw approach}

High aspect ratio nanocrystals were produced using a freezethaw step, indicated in the cryoTEM images of ciprofloxacin liposomes in Fig. 1. Prior to freeze-thaw, and in the absence of
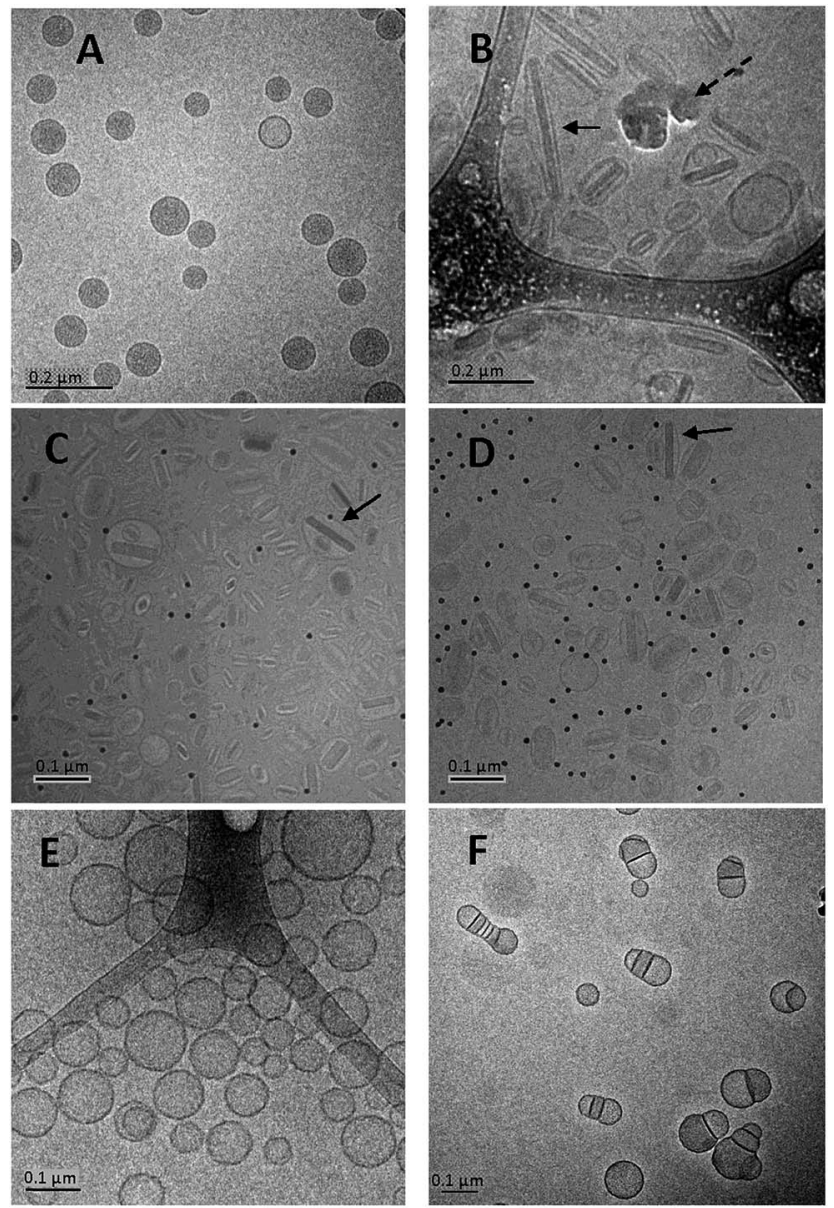

G

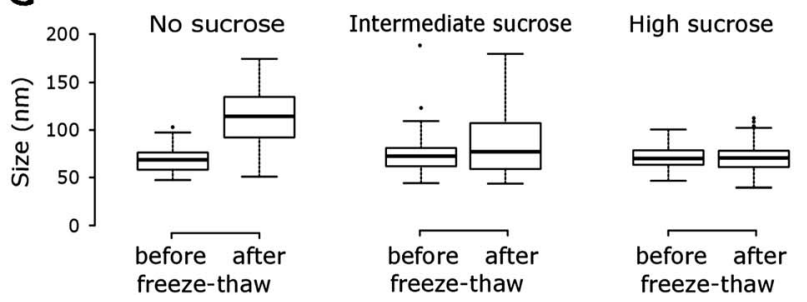

Fig. 1 Effect of internal sucrose concentrations on cryoTEM morphology for liposomes containing ciprofloxacin before and after freeze-thaw. (A) No internal sucrose before freeze-thaw; (B) no internal sucrose after freeze-thaw; (C) intermediate loading of internal sucrose (17.1 $\mathrm{mg} \mathrm{mL}^{-1}$ ) after freeze-thaw; (D) high loading of internal sucrose $\left(51.3 \mathrm{mg} \mathrm{mL}^{-1}\right)$ after freeze-thaw; (E) empty liposomes before freeze-thaw; (F) empty liposomes after freeze-thaw. Drug-containing liposomes were loaded with ciprofloxacin at $12.5 \mathrm{mg} \mathrm{mL}^{-1}$, with external sucrose at $90 \mathrm{mg} \mathrm{mL}^{-1}, \mathrm{pH}$ 6.0. The solid arrow in (B) shows an extremely elongated liposome containing a nanocrystal. The dashed arrow in (B) represents ice artifacts during cryo-TEM imaging. The small black dots in (C) and (D) represent gold fiducials of $14 \mathrm{~nm}$ in size. The solid arrows in (C) and (D) identify liposomes containing a drug nanocrystal as well as a smaller liposome containing its own nanocrystal. The scale bar in the bottom left-hand corner of micrographs (A) and (B) is $200 \mathrm{~nm}$ and in (C, D, E and F) is $100 \mathrm{~nm}$. (G) A box and whisker plot showing the median liposome length, quartile length, and extreme values for each liposome formulation. 
internal sucrose, uniform, unilamellar liposomes were present approximately $70-90 \mathrm{~nm}$ in size (Fig. 1a) in agreement with previous reports. ${ }^{2}$ The liposomes containing intermediate and high internal sucrose concentrations were similar in size, shape and lamellarity. After the freeze-thaw step, the majority of the liposomes contained elongated structures within the vesicles regardless of internal sucrose concentration, consistent with the formation of drug nanocrystals (Fig. 1b-d). The compartmentalization of the drug nanocrystals is clear - some crystals were as large as $200 \mathrm{~nm}$ in length yet were still contained within intact liposomes (Fig. 1b). No drug crystals were apparent outside the liposomes (Fig. 1b-d). Thus the liposomes acted as miniature crystallization reactors, confining the crystal growth within the boundary imposed by the liposomal membrane. There was a small population of liposomes containing a nanocrystal that were together encapsulated inside a larger liposome also containing a separate nanocrystal (solid arrows in Fig. 1c and d).

In additional experiments, it was determined that the external sucrose concentration needed to be above a critical cryoprotectant to lipid ratio of $\sim 2: 1$ (w/w) for the formation of stable liposome dispersions after the freeze-thaw step; otherwise the liposomes precipitated in response to freeze-thaw. Liposomes containing ciprofloxacin $\left(12.5 \mathrm{mg} \mathrm{mL}^{-1}\right)$ prior to freeze-thaw had a similar particle size distribution regardless of internal sucrose concentration, with mean sizes by dynamic light scattering (DLS) ranging from 82 to $91 \mathrm{~nm}$ (Table 1). There was an increase in mean particle size after freeze-thaw for all three formulations that was dependent on the internal sucrose concentration. The largest increase $(\sim 59 \mathrm{~nm})$ was observed for the formulation lacking internal sucrose while the formulation with the highest internal sucrose was the most stable to freezethaw with an increase in mean size of only $\sim 7 \mathrm{~nm}$ (Table 1).

The box and whisker analysis of the liposome Feret length from cryoTEM analysis (Fig. 1g) provides a more direct measure of liposome size than does DLS. Similar to the DLS results, the Feret length measurements also indicated the greatest increase in particle size after freeze-thaw to occur without internal sucrose, with an increase from 68.7 to $114.4 \mathrm{~nm}$ (Table 2). The liposomes without internal sucrose became more elongated (lower roundness) in response to freeze-thaw, stretched by the growth of the internal nanocrystals (Table 2). The liposomes containing the highest internal sucrose concentration again showed the least change in vesicle size (with an increase in median Feret length of only $0.5 \mathrm{~nm}$ ) and a minimal change in roundness (Table 2), consistent with the trend observed by DLS.
Notably, the vesicle size determined from the Feret length in the cryoTEM images (Table 2) differs from the size determined by DLS (Table 1) with cryoTEM imaging indicating smaller liposomes even when characterized by maximum Feret length. This is consistent with previous reports of smaller liposome sizes through analysis by cryoTEM imaging compared to DLS..$^{19}$ DLS is an indirect estimate of vesicle size which measures the hydrodynamic diameter of the vesicles and is influenced by associated ions and surface structure and so generally gives larger sizes than direct measurements like cryoTEM imaging. Quantitation of mean size by cryoTEM imaging can also be confounded by size-sorting and the preferential exclusion of larger particles from the center of the grid where the film is thin. ${ }^{20}$

Empty liposomes containing only external sucrose and no drug were exposed to freeze-thaw with the expectation that freeze-thaw would have no effect on vesicle size. The empty liposomes (Fig. 1e) were of similar size and lamellarity to drugloaded liposomes prior to freeze-thaw (Fig. 1a), but were lighter in shading indicating the absence of encapsulated drug. Unexpectedly, the mean vesicle size for the empty liposomes also increased after freeze-thaw by $\sim 28 \mathrm{~nm}$ (Table 1), with the cryoTEM images indicating the presence of vesicle agglomerates rather than internal crystalline structures (Fig. 1f).

\section{Confirmation of asymmetry of particles in liquid dispersion}

While DLS measurements provide a broad understanding of the size distribution of the particles as approximated spheres, they cannot inform on the asymmetric nature of particles such as those clearly appearing to be stretched under the influence of the encapsulated nanocrystals. It is also necessary to exclude the potential influence of the vitrification step in cryomicroscopy techniques on the behaviour of the system, inducing artefacts that may differ from the initial liquid sample. Therefore SAXS and DDLS were employed to further confirm the asymmetric nature of the particles in dispersion.

The SAXS and DDLS results both indicated the presence of cylindrical particles. Cylinder dimensions were estimated by combining results from SAXS and DDLS. The low $q$ scattering for the liposome sample containing drug nanocrystals showed a $q^{-1}$ dependence, indicative of rod-like particles, consistent with the microscopy images, which was absent from the control sample prior to freeze-thaw (without nanocrystals, Fig. 2).

Further, the wider angle scattering of the dispersions indicated a possible Bragg peak in the sample at $q=0.38 \AA^{-1}$

Table 1 Size distribution of liposomes by dynamic light scattering before and after freeze-thaw

\begin{tabular}{|c|c|c|c|c|c|}
\hline \multirow[b]{2}{*}{ Preparation } & \multicolumn{2}{|c|}{ Sucrose $\left(\mathrm{mg} \mathrm{mL}^{-1}\right)$} & \multicolumn{2}{|c|}{ Mean particle size (nm) [SD] } & \multirow{2}{*}{$\begin{array}{l}\text { Change in mean particle } \\
\text { size }(\mathrm{nm})\end{array}$} \\
\hline & Internal & External & Before freeze/thaw & After freeze/thaw & \\
\hline No internal sucrose & 0 & 90 & $91.0[25.7]$ & $150.0[75.5]$ & 59.0 \\
\hline Intermediate sucrose & 17.1 & 70.7 & $88.6[16.5]$ & $128.8[63.3]$ & 40.2 \\
\hline High sucrose & 51.3 & 70.7 & $82.7[23.2]$ & $89.9[33.6]$ & 7.2 \\
\hline Empty liposomes & 0 & 90 & $92.6[27.6]$ & $121.0[24.4]$ & 28.4 \\
\hline
\end{tabular}


Table 2 Feret length and roundness for liposomes before and after freeze-thaw

\begin{tabular}{|c|c|c|c|c|}
\hline Internal sucrose loading & $\begin{array}{l}\text { Exposure to } \\
\text { freeze-thaw }\end{array}$ & $\begin{array}{l}\text { Median Feret } \\
\text { length (nm) }\end{array}$ & $\begin{array}{l}\text { Median minimum } \\
\text { Feret length }(\mathrm{nm})\end{array}$ & Roundness \\
\hline None & No & 68.7 & 59.9 & 0.87 \\
\hline \multirow[t]{2}{*}{ Intermediate $\left(17.1 \mathrm{mg} \mathrm{mL}^{-1}\right)$} & No & 69.9 & 61.9 & 0.89 \\
\hline & Yes & 74.5 & 57.4 & 0.77 \\
\hline High $\left(51.3 \mathrm{mg} \mathrm{mL}^{-1}\right)$ & No & 67.4 & 58.8 & 0.87 \\
\hline
\end{tabular}

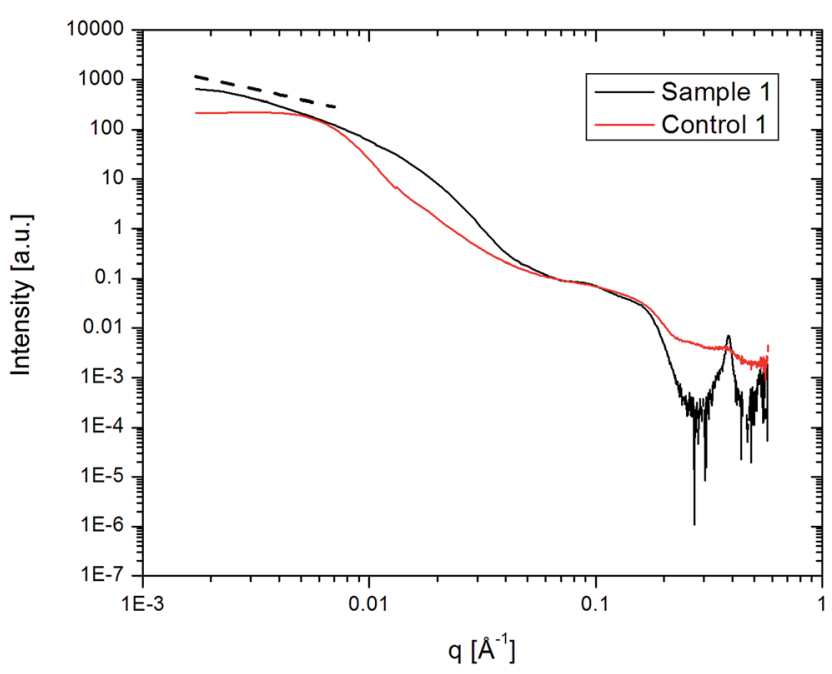

Fig. 2 Merged SAXS curves for liposomes with no internal sucrose before [Control 1] and after freeze-thaw [Sample 1]. The dashed line with slope -1 was calculated to highlight the $q^{-1}$ dependence of the signal at low $q$ characteristic for rod-like particles.

(Fig. 2). While it is not possible under the current configuration to determine the polymorphic form of drug in the nanocrystals, this aspect will be further explored in future studies, as the polymorphic form of the drug is important for solubility and dissolution characteristics.

Using the inverse Fourier transform (IFT) method of modelindependent analysis of the low $q$ scattering data, ${ }^{21}$ the $p(r) v s . r$ pair distance distribution function indicates a particle thickness of approximately $50 \mathrm{~nm}$, and a length beyond the resolution of the SAXS setup, but estimated by extrapolation to be approximately $250 \mathrm{~nm}$ (Fig. 3). These dimensions are overall larger than those indicated by microscopy, but confirm the asymmetry in dimensions, and further work using deuterated lipids and contrast matching neutron scattering to better resolve the crystal from the liposome structure by scattering approaches is planned.

The depolarized dynamic light scattering measurements further supported the presence of anisotropic particles with a linear dependence of the correlation function decay rate with $q^{2}$, as illustrated in ESI Fig. S1 and S2. $\dagger$

\section{Confirmation of drug nanocrystal encapsulation inside liposomes}

CryoTEM only provides a two-dimensional projection of the drug nanocrystals, and would not differentiate nanocrystals adsorbed or associated with the outside of the liposomes from truly encapsulated particles. Cryo-electron tomography was

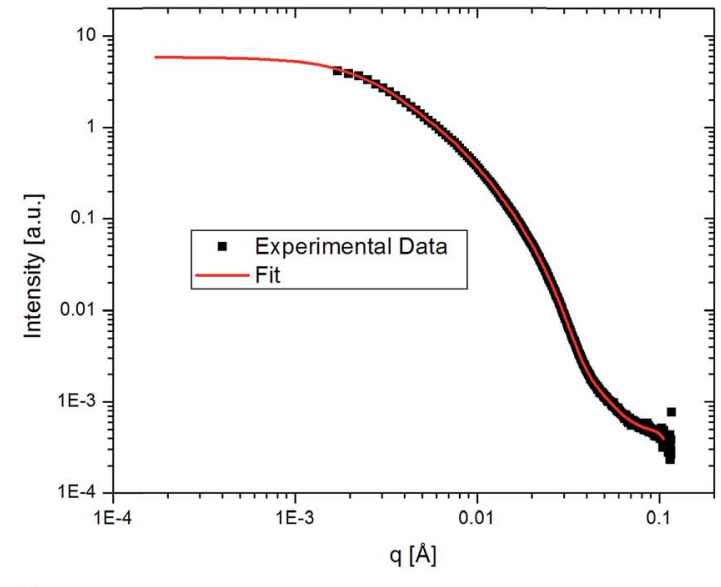

a

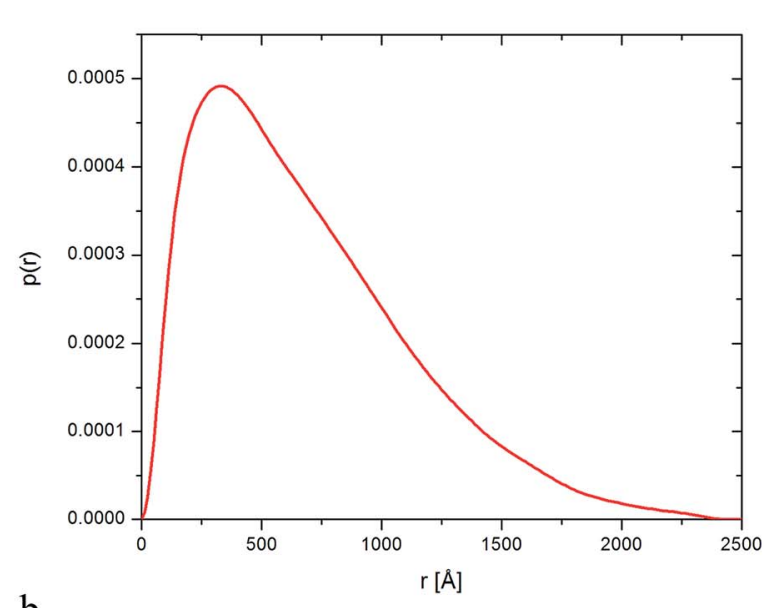

b

Fig. 3 Liposomes with no internal sucrose after freeze-thaw (Sample 1). Experimental data and model independent fit calculated with the IFT method extrapolated to low $q$ in (a). Pair distance distribution function calculated by Fourier transformation of the data in (b). The shape is characteristic for cylinders. Yet, the maximum dimension is above the resolution limit of the SAXS set-up (around $250 \mathrm{~nm}$ extrapolated by calculation). Of interest here is the cylinder cross-section dimension ( $50 \mathrm{~nm})$. 
therefore used to confirm that the nanocrystals indeed resided within the host liposome structures. The three orthogonal views (Fig. 4) of representative 3D reconstructed liposomes confirm that the nanocrystals coincide with the liposomes and are hence intravesicular structures. In addition, the absolute absence of such nanocrystals outside the liposomes clearly confirms that the nano-compartmentalization is exclusive and controls the location of crystallization. Encapsulation of the nanocrystals was confirmed for all three formulations although there was a variation in range of vesicle shapes and nanocrystal lengths. As mentioned for cryoTEM, most liposomes contained only one nanocrystal, however there was a minority of liposomes $(<10 \%)$ which contained both a nanocrystal and another liposome containing its own nanocrystal. The 3D stacks show that these encapsulated liposomes are indeed captured inside a larger liposome and not merely overlapping in the axial direction.

The 3D stacked data presentation, available as videos in the $\mathrm{ESI}, \uparrow$ confirms that the liposomes are intact and there is a random angular distribution of nanocrystals within the population of liposomes. Such a lack of preferential orientation suggests that the nanocrystals grow in each liposome without external influence of nearby nanocrystal growth.

\section{Formation of nanocrystals reduces the drug release rate from liposomes}

Compartmentalization of the drug nanocrystals inside liposomes was anticipated to slow dissolution of the crystalline drug due to a non-sink environment inside the liposomes. Release experiments were conducted both before and after freeze-thaw of the formulations containing increasing concentrations of internal sucrose (Fig. 5a-c). After freezethaw, all three formulations had a slower release profile compared to the untreated control samples, consistent with the presence of nanocrystalline drug isolated inside a non-sink compartment of the liposome. The initial rates of release, defined by the percent release at $30 \mathrm{~min}$, were consistently slower $(\sim 59 \%, \sim 48 \%$ and $\sim 36 \%$ slower, respectively) for the three formulations compared to their respective untreated controls. Thus the in vitro release in Fig. 5 supports the inference that the drug has been converted to crystalline form in the interior of the liposomes and provides a new ratelimiting step for the ciprofloxacin release, i.e., dissolution. The liposomes with no internal sucrose showed the greatest reduction in the drug release rate (Fig. 5a) while the liposomes with the highest internal sucrose concentration showed the least reduction in release rate (Fig. $5 \mathrm{c}$ ). This release rate data is consistent with the observed increase in vesicle size upon freeze-thaw (Tables 1 and 2). Vesicles containing larger crystals, described by a smaller ratio of total crystal surface area to crystal volume, would be expected to have slower release rates and this was indeed observed. The system with the largest increase in liposome size after freeze/thaw had the slowest release rate (Fig. 5a) and vice versa for the smallest increase in liposome size (Fig. 5c) had the fastest release rate.
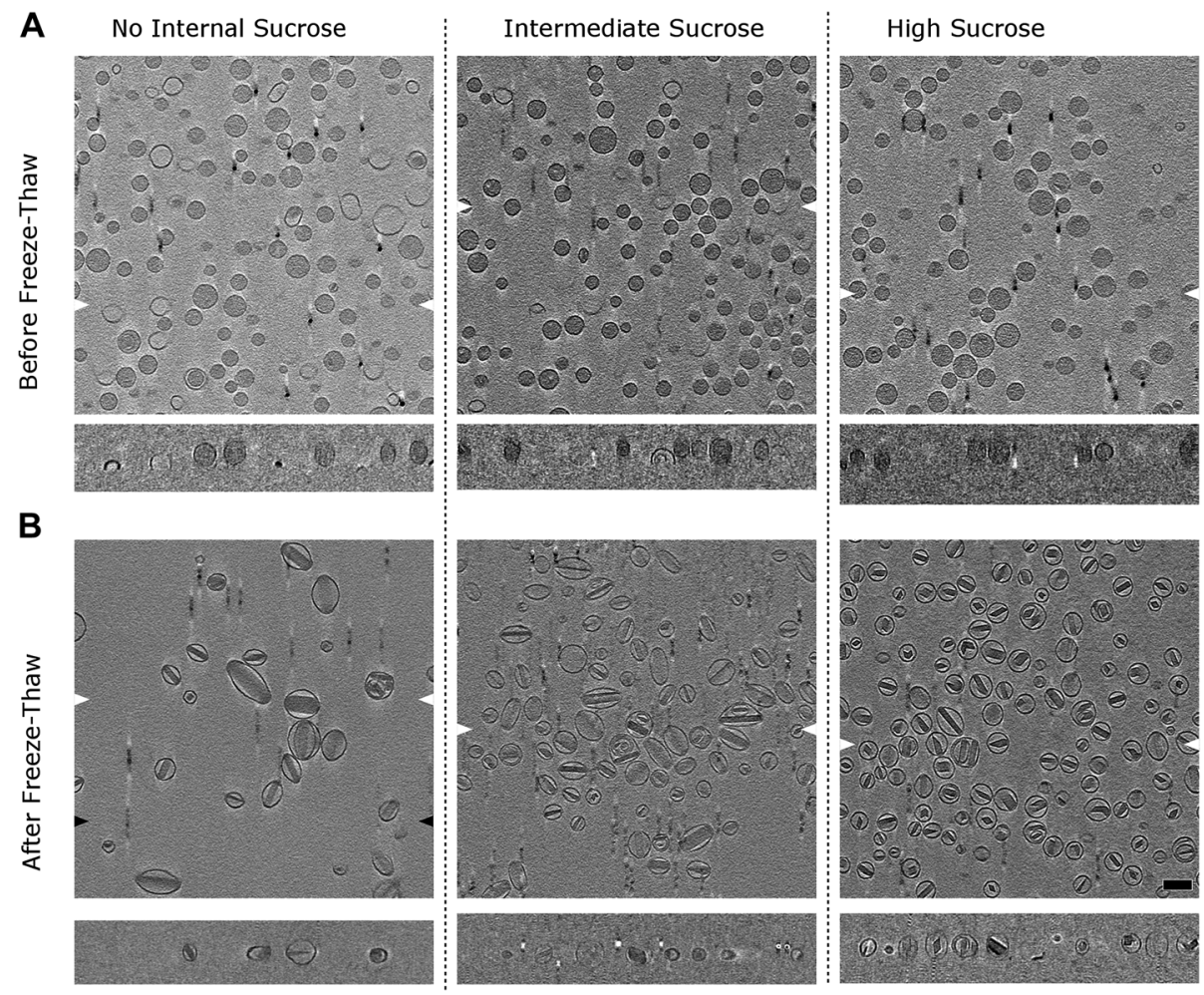

Fig. 4 Cryo-electron tomography, before and after freeze-thaw of ciprofloxacin-loaded liposomes. The scale bar is $100 \mathrm{~nm}$. (a) No internal sucrose before freeze-thaw; (b) no internal sucrose after freeze-thaw; (c) intermediate loading of internal sucrose before freeze-thaw; (d) intermediate loading of internal sucrose after freeze-thaw; (e) high loading of internal sucrose before freeze-thaw; (f) high loading of internal sucrose after freeze-thaw. The small black dots represent gold fiducials of $14 \mathrm{~nm}$ in size. 

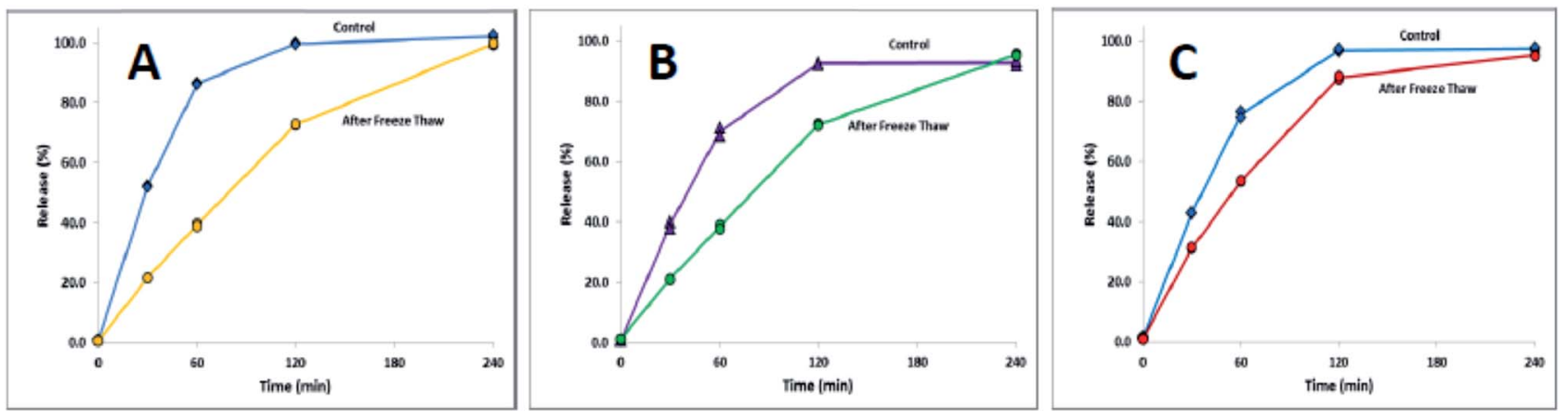

Fig. 5 The effect of the freeze-thaw process on the in vitro release profiles of ciprofloxacin from three liposomal formulations with increasing concentration of internal sucrose. (A) No internal sucrose, (B) $17.1 \mathrm{mg} \mathrm{mL}^{-1}$ internal sucrose, (C) $51.3 \mathrm{mg} \mathrm{mL}^{-1}$ internal sucrose. The formulations (containing ciprofloxacin at $12.5 \mathrm{mg} \mathrm{mL}^{-1}$ ) were diluted to $50 \mu \mathrm{g} \mathrm{mL}^{-1}$ ciprofloxacin in HEPES buffered saline (HBS) prior to a $1: 1$ dilution in bovine serum to stimulate the release of ciprofloxacin after incubation at $37^{\circ} \mathrm{C}$ for up to $4 \mathrm{~h}$. Duplicate samples were analyzed at each time point.

Similarity analysis $\left(f_{2}\right)$ was also used to quantify the apparent visual differences in release rates and the results are tabulated in ESI (Tables S1 and S2†). For the formulation without internal sucrose, the similarity factor was 27.7 , much less than the critical value of 50 , confirming that there is a real difference between the release profile after freeze-thaw compared to that prior to freeze-thaw. For the liposomes containing internal sucrose, the similarity factors were less than 50 when the in vitro release profile prior to freeze-thaw was compared to that after freeze-thaw, again indicating a significant difference. As a control, the profiles for the liposome formulations containing internal sucrose were compared to those for liposome formulations lacking internal sucrose. In both cases the similarity factors were greater than 50 indicating that there was not a meaningful difference between the three control formulations (Tables S1 and S2 $\dagger$ ).

\section{Discussion of mechanism of nanocrystal formation and implications for treating lung infections}

The discovery of this simple process of forming drug nanocrystals arose from a need to modulate the rate of release of ciprofloxacin from liposomes intended for pulmonary drug delivery applications, by transformation of its physical state without a change in composition. The primary rate controlling membrane, i.e., the liposome bilayer, would ideally remain unperturbed on formation of the nanocrystal, and act to prevent aggregation of crystals or Ostwald ripening. This indeed appeared successful with all nanocrystals clearly encapsulated by and limited in growth due to the bilayer envelope, and provides a potential simple route to formation of drug nanocrystals not previously identified.

The asymmetric shape of the crystals, evident from cryoTEM imaging and confirmed by the scattering results, strongly indicates that the drug indeed precipitates in a crystalline, rather than amorphous solid state form, growing from the preferred crystal face as is the case in bulk crystallization processes. In consideration of the aim of slowing dissolution, this is a more optimal outcome, as thermodynamically favoured crystalline forms will have reduced solubility in the interior of the liposomes compared to high energy amorphous materials. The solid state form of the drug inside the liposomes has not yet been determined but will be the subject of future studies: WAXS will be used to determine whether a co-existing halo underlies the crystalline diffraction pattern which would indicate the presence of amorphous drug in the liposomes, as well as reveal the presence of other polymorphic crystalline structures. While visually there does not appear to be 'non-crystalline' solid mass in the liposomes, this does not exclude the possibility of coexisting amorphous material, although its presence is expected to be low in any case. It was reported for another liposomal formulation of ciprofloxacin that the ciprofloxacin selfassociated but did not precipitate out of solution, even though the solubility limit was exceeded by two orders of magnitude, ${ }^{8}$ suggesting that an amorphous solid-state form is unlikely.

For the drug nanocrystals to precipitate from the concentrated internal solution of the liposomes, nucleation precedes crystal growth. We hypothesize that small ice particles formed during thawing within the liposome vesicles could have provided nucleation sites for the low solubility solid form of ciprofloxacin. Interestingly, the vast majority of liposomes each contained one, and only one, drug nanocrystal (Fig. 1). There are a number of implications of this 'one nanocrystal per liposome' observation. Firstly, the initiation of crystal formation must be a nearly homogeneous phenomenon under these conditions (i.e., it occurs in every liposome). Second, the majority of liposomes must be stable to the crystal growth process and able to accommodate crystals which can become very elongated, often more than $200 \mathrm{~nm}$ in length. The absence of external ciprofloxacin is also supported by data from the in vitro release assay (Fig. $5 \mathrm{a}-\mathrm{c}$ ) showing the absence of unencapsulated drug at the $T=0$ time point. This result confirms that very few if any liposomes were breached during the process which would otherwise lead to substantial amounts of unencapsulated drug. Third, either a single intra-liposome crystal nucleation event occurs within each liposome during the thawing process, or alternatively, if more than one nucleation event occurs within a vesicle, then one of the crystals rapidly grows at the expense of the other nascent crystals, as would be 
anticipated through Ostwald ripening. In any case, as mentioned above it is clear that inter-liposome Ostwald ripening does not occur, as this would lead to a small number of very large crystals and a large number of apparently empty liposomes. The cryoTEM sample preparation and subsequent analysis was performed on samples immediately after freezethaw suggesting that the rate of crystal growth within each liposome is very fast, occurring within seconds of thawing.

Liposomes are not typically subjected to freezing unless they are intended to be supplied in a lyophilized powder form for reconstitution before use. The freezing introduces ice crystals which can damage the structures leading to aggregation and phase separation. In order to protect the liposomes from such phenomena, during the freeze-thaw process here, it was first necessary to stabilize the liposomes to freeze-thaw. The addition of cryoprotectants; e.g., sucrose and trehalose, has long been known to effectively stabilize some liposomal preparations during freeze-thaw, ${ }^{\mathbf{1 3 - 1 5}}$ or lyophilization. ${ }^{22}$ Cryoprotectants limit mechanical damage and rupture of the lipid bilayer caused by ice crystals during freeze-thaw or the freeze-drying and subsequent rehydration process by maintaining the membrane in a flexible state, by adding bulk to the solution to prevent direct contact between vesicles and by reducing the mobility of the vesicles. ${ }^{23-25}$ The sugar molecules can form hydrogen bonds with the liposome and thus 'replace' the water molecules around the liposomes.

The presence of sucrose within the liposomes led to shorter nanocrystals and less elongated liposomes. While these studies have focused on the effect of sucrose, trehalose was also found to be partially effective, but not as effective as sucrose. CryoTEM images confirmed the presence of nanocrystals in liposomes formulated with trehalose but the liposomes were very elongated and included multilamellar and liposome-encapsulated liposome structures (data not shown). It is unclear why sucrose is superior to trehalose in this regard but it may be related to its ability to hydrogen bond to the specific lipid used in these preparations (hydrogenated soy phosphatidylcholine).

The ramifications of this novel, previously unreported method of incorporation of drug nanocrystals into liposomes may be significant. The in vitro release data showed that in the presence of nanocrystals the release profiles exhibited a statistically significant decrease in release rate compared to that of the unfrozen controls. The IVR assay ${ }^{\mathbf{1 8}}$ was designed as an analytical tool to allow for comparisons to be made between formulations within a reasonable time frame ( $<4$ hours), much shorter than the in vivo half-life of $10.5 \mathrm{~h}$ reported for the control liposomal ciprofloxacin formulation in human subjects after inhalation. ${ }^{\mathbf{1 1}}$ Based on the in vitro release data, the nanocrystalline formulations might be expected to possess an in vivo half-life much longer than $10.5 \mathrm{~h}$; however, this remains to be confirmed in future studies.

We have previously advanced the goal of personalizing liposomal formulations to individualize therapy through increasing the release rate of a liposomal formulation of ciprofloxacin by addition of surfactant under hyperosmotic conditions. ${ }^{2}$ In this paper we report a novel methodology to reduce the release rate of ciprofloxacin from liposomes, through the introduction of a rate-limiting dissolution step, by transforming the encapsulated drug within the liposomes into nanocrystals. Thus, there are now methods available to be appropriately used in the application of liposomes to increase, or decrease, the release rate of drug from these therapeutic nanocarriers. Liposomal ciprofloxacin is being developed as an inhaled therapy to treat lung infections. ${ }^{\mathbf{1 , 1 1 , 1 2}}$ The release profile of the current liposomal ciprofloxacin product in development, Pulmaquin ${ }^{\circledR}$, combines immediately available unencapsulated ciprofloxacin solution with liposomally encapsulated ciprofloxacin that appears to be appropriate for once daily dosing (10.5 h half-life) in non-cystic fibrosis (CF) bronchiectasis patients colonized with infections with Pseudomonas aeruginosa. ${ }^{\mathbf{1 1 , 1 2}}$ Other patient populations may need different release profiles for optimum therapeutic outcomes. For patients with intracellular infections in their alveolar macrophages; e.g., non TB mycobacteria, ${ }^{\mathbf{1}}$ it may be desirable to delay drug release until the macrophages have time to take up the liposomes. Inhalation therapy of a liposomal formulation containing drug nanocrystals might therefore be more efficacious for this patient population.

\section{Experimental section}

\section{Materials}

Liposomes containing $50 \mathrm{mg} \mathrm{mL}^{-1}$ ciprofloxacin with $0,17.1$ or $51.3 \mathrm{mg} \mathrm{mL}^{-1}$ internal sucrose, and empty liposomes, both in a pH 6.0 histidine-buffered aqueous formulation were produced by Northern Lipids Incorporated (Burnaby, BC, Canada) (the ciprofloxacin concentrations are expressed in terms of ciprofloxacin hydrochloride). The following materials were utilized: Nanosep centrifugal filtration devices, $10 \mathrm{k}$ and $30 \mathrm{k}$ molecular weight (Pall Corporation (Ann Arbor, MI)), Donor Adult Bovine Serum (HyClone (Logan, Utah)), sucrose (Sigma-Aldrich (St. Louis, MO)), HEPES, free acid (Avantor (Center Valley, PA)), sodium chloride (Amresco (Solon, OH)), HPLC grade methanol (Fisher Scientific (Fair Lawn, NJ)) and triethylamine (TEA, JT Baker (USA)). Deionized water was used for all studies.

\section{Preparation of liposomal ciprofloxacin formulations}

The preparation of ciprofloxacin loaded liposomes containing cholesterol and hydrogenated soy phosphatidylcholine (HSPC) has been reported previously. ${ }^{2,19}$ Briefly, multilamellar liposomes were extruded through membranes to produce unilamellar liposomes which were then diafiltered directly to produce empty liposomes, or actively loaded with ciprofloxacin. ${ }^{19,26}$ Unencapsulated ciprofloxacin was removed by diafiltration resulting in $>99 \%$ encapsulated ciprofloxacin at a target concentration of $50 \mathrm{mg} \mathrm{mL} \mathrm{m}^{-1}$.

\section{Freeze-thaw studies to create nanocrystals}

Ciprofloxacin liposomes at $50 \mathrm{mg} \mathrm{mL} \mathrm{m}^{-1}$ were diluted four-fold with $180 \mathrm{mg} \mathrm{mL}{ }^{-1}$ sucrose and water to produce liposomes containing ciprofloxacin at $12.5 \mathrm{mg} \mathrm{mL}^{-1}$. These dispersions were transferred in $1 \mathrm{~mL}$ aliquots to either plastic Eppendorf tubes or HPLC glass vials for freeze-thaw evaluation. The 
formulations were frozen either in liquid nitrogen or by storing for a minimum of $20 \mathrm{~h}$ in a $-50{ }^{\circ} \mathrm{C}$ freezer. The samples were thawed in room temperature water and vortexed to ensure homogeneity prior to subsequent evaluation of physical appearance, vesicle size, drug encapsulation, in vitro release, cryoTEM analysis, small angle X-ray scattering or (depolarised) dynamic light scattering. Following thawing, formulations either retained visual clarity indicating stability to freeze-thaw, or the presence of precipitated matter representing agglomerated vesicles if the formulation was not stable to freeze-thaw.

\section{Dynamic light scattering}

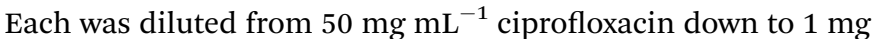
$\mathrm{mL}^{-1}$ with saline, and $0.5 \mathrm{~mL}$ was transferred to a disposable culture tube (Kimble Glass Inc., USA) for size analysis using a Submicron Particle Sizer Autodilute Model 370 (Nicomp, USA). The following instrument parameters were selected: temperature: $23{ }^{\circ} \mathrm{C}$; viscosity: 0.933 ; refractive index: 1.333; intensity set point: $300 \mathrm{kHz}$; channel width: $10 \mu \mathrm{s}$; scattering angle: 90; run time: 5 min; mode: vesicle; Gaussian distribution. The mean and standard deviation (SD) of the particle size distribution were recorded.

\section{Assessment of drug encapsulation}

Nanosep Omega centrifugation devices (Pall Corporation, Ann Arbor, MI) with modified polyethersulfone membrane filters of 10000 or 30000 molecular weight cut-offs were used to separate free drug from liposomal encapsulated drug. Each sample was diluted twenty-fold into acetate buffer ( $50 \mathrm{mM}$ sodium acetate, $145 \mathrm{mM} \mathrm{NaCl}, \mathrm{pH} 4.0$ ) and $400 \mu \mathrm{L}$ was transferred to the centrifugation device and centrifuged for $10 \mathrm{~min}$ at $10000 \mathrm{rpm}$ $(8100 \mathrm{~g})$. A centrifugation time of $10 \mathrm{~min}$ and $8100 \mathrm{~g}$ was chosen to ensure that there was adequate volume in the filtrate to transfer to HPLC vials without risk of rupture to the membranes due to too high or prolonged exposure to the centrifugation force. These conditions were previously shown to provide quantitative recovery. ${ }^{18}$ The filtrate, representing the free ciprofloxacin component, was analysed by HPLC for ciprofloxacin content. The total amount of ciprofloxacin, representing both the encapsulated and free drug, was quantified by HPLC after diluting the liposome sample twenty-fold into $80 \%$ methanol to solubilize the liposomes. The percent encapsulation was determined by comparing the free drug to the total drug in each sample.

\section{Cryogenic transmission electron microscopy (cryoTEM)}

CryoTEM analysis was used to obtain visual images of the liposomal ciprofloxacin and drug-free liposomal dispersions and to confirm the presence or absence of drug nanocrystals, either prior to or after freeze-thaw. A JEOL 2100 (Tokyo, Japan) instrument operated at $200 \mathrm{kV}$ or a Tecnai Spirit transmission electron microscope (FEI (Hillsboro, Oregon)) operated at 120 $\mathrm{kV}$ was used. For the imaging done on the JEOL 2100 instrument, the control or thawed drug-free liposome samples were diluted ten-fold with water and $3 \mu \mathrm{L}$ of each sample was applied to a glow discharge Quantifoil carbon grid (Jena, Germany) in a chamber controlled to $22{ }^{\circ} \mathrm{C}$ and $100 \% \mathrm{RH}$. Grids were blotted once with filter paper, at a blotting angle of $2 \mathrm{~mm}$ for $2 \mathrm{~s}$, and vitrified by plunging into liquid ethane using a Vitrobot (F.E.I., Eindhoven, Netherlands). The vitrified samples were stored in liquid nitrogen prior to cryoTEM analysis. For the imaging done on the Tecnai Spirit instrument, the control and freeze-thawed samples were prepared and analysed as described below in the cryo-electron tomography section.

The length of the control liposomes or liposomes containing nanocrystals was analysed manually using Image J software ${ }^{27}$ and data presented is representative of more than 50 individual measurements on at least 10 different micrographs (mean \pm $\mathrm{SD})$. Both the maximum and minimum Feret length for each vesicle were measured between two points lying on the liposome membrane across from each other. From these numbers, the roundness $(R)$ of each vesicle can be calculated as the ratio of the median minimum Feret length to the median Feret length, where a value of 1 represents a perfect sphere and a value of 0 represents an infinite line.

\section{Cryo-electron tomography}

Cryo-electron tomography, as described previously, ${ }^{28}$ was conducted to provide additional detail on the size and shape of the drug nanocrystals within the liposomes. Briefly, tomography was performed either prior to freezing (control) or after freezethaw using a Tecnai Spirit transmission electron microscope (FEI (Hillsboro, Oregon)) operated at $120 \mathrm{kV}$. Gold nanoparticle (14 nm) fiducials ${ }^{29}$ at a final concentration of $\sim 25 \mu \mathrm{g} \mathrm{mL}{ }^{-1}$ were coated with bovine serum albumin (Sigma-Aldrich (St. Louis, MO)) and mixed with the liposome samples at a final concentration of $\sim 5 \mathrm{mg} \mathrm{mL}^{-1}$ (ten-fold dilution of initial preparation). Each sample $(5 \mu \mathrm{L})$ was applied to a holey carbon-coated copper grid (200 mesh, Plano AG (Germany)). Grids were blotted once with filter paper for 4-5 $\mathrm{s}$ and vitrified by plunging into liquid ethane. The vitrified samples were stored in liquid nitrogen prior to use, and then mounted in a Gatan 626 cryo-holder (Gatan, Inc. (Pasadena, CA, USA)) for cryo-electron tomography. Images were recorded with an Eagle camera (FEI, $4096 \times$ 4096 pixels) under low-dose conditions using SerialEM. ${ }^{30}$ Sample holder tilting angles ranged from -60 to 60 degrees with increments of either 2 or 3 degrees. The defocusing setting was $-4.0 \mu \mathrm{m}$. After reconstruction, approximately 200 slices of $\sim 1.5 \mathrm{~nm}$ thickness each were obtained.

\section{Small angle X-ray scattering (SAXS) measurements}

SAXS measurements were performed at the SAXS/WAXS beamline at the Australian Synchrotron. ${ }^{31}$ An X-ray beam with a wavelength of $1.12 \AA$ was used. A sample to detector distance of $567 \mathrm{~cm}$ and $7 \mathrm{~m}$ gave the combined $q$-range $0.002<q<1.7$ $\AA^{-1}$, where $q$ is the length of the scattering vector, defined by $q=$ $(4 \pi / \lambda) \sin (\theta / 2), \lambda$ is the wavelength and $\theta$ the scattering angle. The 2D SAXS patterns were acquired within $1 \mathrm{~s}$ using a Pilatus 1 M detector with active area $169 \times 179 \mathrm{~mm}^{2}$ and with a pixel size of $172 \mu \mathrm{m}$. All experiments were performed at $T=27{ }^{\circ} \mathrm{C}$ (hutch temperature at the beamline). For each curve, multiple frames were recorded and averaged manually after inspection for beam 
damage; no beam damage was observed in the samples. Two dimensional scattering patterns were integrated into the onedimensional scattering function $I(q)$ using the in-house developed software package scatterBrain. Scattering curves are plotted as a function of relative intensity, I versus $q$. Water was subtracted as background from all scattering curves and Porod analysis was used to determine the constant background that was then subtracted from the data.

\section{SAXS data analysis}

The generalized indirect Fourier transformation method (GIFT) was used for a model free analysis of the scattering data. ${ }^{32-34}$ For particles of arbitrary shape with an electron density difference of $\Delta \rho(r)$ relative to the mean value, the pair-distance distribution function $p(r)$ is given by $p(r)=r^{2} \Delta \tilde{\rho}^{2}(r)$ where $\Delta \tilde{\rho}^{2}(r)$ is the convolution square of the electron density averaged for all directions in space. This averaging causes no loss of information in the case of particles with spherical symmetry.

The $p(r)$ is calculated from the scattered intensity $I(q)$ using the following equation: ${ }^{21}$

$$
I(q)=4 \pi \int_{0}^{\infty} p(r) \frac{\sin (q r)}{q r} \mathrm{~d} r
$$

and gives a real space representation of the overall shape of the particles. ${ }^{35}$ In the case of spherical geometry, deconvolution of the $p(r)$ gives the radial contrast profile $\Delta \rho(r)$ in electron density relative to the mean value, which gives information about the internal structure of the scattering particles. ${ }^{36}$ In the case of cylindrical micelles with sufficient length/diameter ratio $(>3)$, the cross section can be investigated using the cross section pair distance distribution function $p_{\mathrm{c}}(r)$. The radial electron density profile $\Delta \rho_{\mathrm{c}}(r)$ is related to the $p_{\mathrm{c}}(r)$ via $p_{\mathrm{c}}(r)=r \Delta \tilde{\rho}^{2}(r)$.

The $p_{\mathrm{c}}(r)$ can be calculated from $I(q)$ with the following equation:

$$
I(q)=\frac{2 \pi^{2} L}{q} \int_{0}^{\infty} p_{\mathrm{c}}(r) J_{0}(q r) \mathrm{d} r
$$

In this equation, $J_{0}(q r)$ is the zero-order Bessel function and $L$ is the length of the cylinder.

Deconvolution of $p(r)$ for spherical particles and $p_{c}(r)$ for cylindrical micelles gives the radial contrast profile $\Delta \rho(r)$ or $\Delta \rho_{\mathrm{c}}(r)$ in electron density or scattering length density, which gives information about the internal structure of the scattering particles.

\section{(Depolarised) dynamic light scattering ((D)DLS)}

DLS and DDLS measurements were performed on an ALV-5022F spectrometer, using a vertically polarized Helium Neon laser (wavelength $633 \mathrm{~nm}$ ). The scattered light passed through a crossed polarizer, which was carefully adjusted to achieve minimum scattered intensity for the DDLS measurements. Correlation functions were collected at different scattering angles between $20^{\circ}$ and $90^{\circ}$. At each angle, ten measurements of 1 min duration were performed, and the average of the data sets was taken for each angle. DDLS takes advantage of the fact that the light scattered from optically homogeneous spherical particles maintains the same polarization as the incident light. However, optically anisotropic particles also scatter light. The correlation function measured in DDLS is a combination of translational diffusion and rotational diffusion, and the decay rate of the depolarized field correlation function, $\Gamma$, is given by: $\Gamma=q^{2} D_{\mathrm{T}}+6 D_{\mathrm{R}}$ where $q$ was determined from the correlation function by cumulant expansion, $D_{\mathrm{T}}$ is the translational diffusion coefficient and $D_{\mathrm{R}}$ is the rotational diffusion coefficient. ${ }^{37}$

\section{In vitro release assay of ciprofloxacin from liposomes}

The in vitro release assay measures the rate and extent of release of ciprofloxacin from liposomes. ${ }^{18}$ Briefly, the liposomal samples were diluted to $50 \mu \mathrm{g} \mathrm{mL}^{-1}$ ciprofloxacin in HEPES Buffered Saline (HBS: $20 \mathrm{mM}$ HEPES, $145 \mathrm{mM} \mathrm{NaCl}, \mathrm{pH} 7.4)$ and mixed one-to-one with chilled $\left(2-8{ }^{\circ} \mathrm{C}\right)$ bovine serum (Hyclone) and placed in a shaking water bath (Techne, TSBS40 (Staffordshire, UK)) at $37{ }^{\circ} \mathrm{C}$ and $150 \mathrm{rpm}$. Duplicate samples were removed after incubation for 30,60, 120 and $240 \mathrm{~min}$, diluted $1: 1$ with chilled $\left(2-8{ }^{\circ} \mathrm{C}\right) \mathrm{HBS}$ and placed in an ice-water bath to terminate any further release of encapsulated drug from the liposomes. The released ciprofloxacin was separated from the liposome-encapsulated ciprofloxacin by transferring $400 \mu \mathrm{L}$ of each chilled sample to a Nanosep centrifugal device and centrifuging at $10000 \mathrm{rpm}$ for $10 \mathrm{~min}$. The filtrate was removed for subsequent quantitation of the released ciprofloxacin by HPLC. This value was normalized by dividing by 0.93 , to correct for a small but reproducible loss of free drug in the filtration devices in the presence of serum. ${ }^{18}$ The original liposomal sample was diluted into $80 \%$ methanol to dissolve the liposomes and allow for quantitation of the total amount of ciprofloxacin by HPLC. The percent release at each time point was calculated by comparing the free drug to the total drug. The initial rate of release, $T_{30} \mathrm{~min}^{-} T_{0 \mathrm{~min}}$, was defined as the amount of released drug at the $30 \mathrm{~min}$ time point minus that present at the initial time point.

To determine whether there was a statistically meaningful change in the in vitro release profiles of the liposomal formulations before and after freeze-thaw, similarity factor $\left(f_{2}\right)$ analysis was used. This methodology is advocated by the regulatory authorities to compare dissolution profiles for modified release solid oral dosage forms ${ }^{38}$ but is also generally recommended for liposomal products. The similarity factor is calculated by measuring the difference between the mean in vitro release values of a test and reference product at each of the time points. A similarity factor that is greater than 50 indicates the profiles are similar while a value less than 50 implies they are different. A value of 100 represents an identical test and reference profile. $^{38}$

\section{High performance liquid chromatography (HPLC) assay of ciprofloxacin}

The amount of ciprofloxacin in each sample was quantified using an HPLC method as described previously. ${ }^{2,18}$ Briefly, HPLC analysis was performed using a Nucleosil C-18 column $(5 \mu \mathrm{m}, 4.6 \times 150 \mathrm{~mm}$, Canadian Life Science, CA) protected with 
a Nucleosil C-18 guard column $(4 \times 3.0 \mathrm{~mm}$, Phenomenex, USA $)$ both at $35{ }^{\circ} \mathrm{C}$. The mobile phase was a mixture of $0.5 \%$ TEA in water, pH 3.0 and $100 \%$ methanol $(83: 17 \mathrm{v} / \mathrm{v})$ and the isocratic elution was performed at a flow rate of $0.9 \mathrm{~mL} \mathrm{~min}^{-1}$. Ciprofloxacin was detected and quantified at a wavelength of $277 \mathrm{~nm}$.

\section{Conclusions}

A new, simple and industrially applicable process to induce nanocrystallization of drug within the confinement of the interior of liposomes is presented. The simple freeze-thaw step results in the formation of a single drug nanocrystal within the vesicles. The length of the drug nanocrystals, and thus the elongation of the liposomes, was reduced with increasing sucrose within the liposomes. The elongation of the liposomes observed by cryoTEM was confirmed using X-ray and light scattering techniques. The nanocrystals were confirmed to reside within the liposomes, rather than adhered to the exterior using cryo-electron tomography. Functionally, and importantly in the context of pulmonary drug delivery, the presence of the drug nanocrystals introduced a rate-limiting dissolution step to release, which resulted in up to a $64 \%$ slower release rate for drug compared to the untreated control formulation. This innovation has the potential to advance inhaled anti-infective therapy by providing a simple methodology that allows the antibiotic release profile to be modified to meet the needs of a specific patient population, and potentially even 'fine-tune' the profile for the needs of each individual patient at the point of care.

\section{Conflict of interest}

David Cipolla, Huiying Wu and Igor Gonda are employees of Aradigm Corporation. Aradigm Corporation is developing inhaled formulations of liposomal ciprofloxacin to treat lung disease.

\section{Acknowledgements}

The SAXS/WAXS studies were conducted at the Australian Synchrotron, Clayton, Victoria, Australia. The DDLS studies were conducted at RMIT University, Melbourne, Australia with the help of Gary Bryant and assistance of Reece Nixon-Luke. The cryo-electron tomography work was supported by the Adolphe Merkle Foundation. The authors also acknowledge the technical assistance of Judy Loo and Delfine Cheng and the Australian Microscopy and Microanalysis Research Facility at the Electron Microscope Unit, The University of Sydney. David Cipolla was a recipient of the Australian Postgraduate Award and the Australian IPRS scholarship.

\section{Notes and references}

1 J. Blanchard, L. Danelishvili, I. Gonda and L. Bermudez, ATS Conference, \#57372, 2014.

2 D. Cipolla, H. Wu, S. Eastman, T. Redelmeier, I. Gonda and H.-K. Chan, J. Pharm. Sci., 2014, 103, 1851-1862.
3 D. D. Lasic, P. M. Frederick, M. C. A. Stuart, Y. Barenholz and T. J. McIntosh, FEBS Lett., 1992, 312, 255-258.

4 D. D. Lasic, B. Ceh, M. C. Stuart, L. Guo, P. M. Frederik and Y. Barenholz, Biochim. Biophys. Acta, 1995, 1239, 145-156.

5 X. Li, D. J. Hirsh, D. Cabral-Lilly, A. Zirkel, S. M. Gruner, A. S. Janoff and W. R. Perkins, Biochim. Biophys. Acta, 1998, 1415, 23-40.

6 S. A. Abraham, K. Edwards, G. Karlsson, N. Hudon, L. D. Mayer and M. B. Bally, J. Controlled Release, 2004, 96, 449-461.

7 I. V. Zhigaltsev, N. Maurer, K. Edwards, G. Karlsson and P. R. Cullis, J. Controlled Release, 2006, 110, 378-386.

8 N. Maurer, K. F. Wong, M. J. Hope and P. R. Cullis, Biochim. Biophys. Acta, 1998, 1374, 9-20.

9 E. Maurer-Spurej, K. F. Wong, N. Maurer, D. B. Fenske and P. R. Cullis, Biochim. Biophys. Acta, 1999, 1416, 1-10.

10 D. C. Drummond, C. O. Noble, M. E. Hayes, J. W. Park and D. B. Kirpotin, J. Pharm. Sci., 2008, 97, 4696-4740.

11 P. Bruinenberg, J. D. Blanchard, D. C. Cipolla, F. Dayton, S. Mudumba and I. Gonda, Respiratory Drug Delivery 2010, ed. R. N. Dalby, P. R. Byron, J. Peart, J. D. Suman, S. J. Farr and P. M. Young, Davis Healthcare Int'l Publishing, River Grove, 2010, pp. 73-81.

12 D. J. Serisier, D. Bilton, A. De Soyza, P. J. Thompson, J. Kolbe, H. W. Greville, D. Cipolla, P. Bruinenberg and I. Gonda, Thorax, 2013, 68, 812-817.

13 G. Strauss and H. Hauser, Proc. Natl. Acad. Sci. U. S. A., 1986, 83, 2422-2426.

14 M. Ausborn, H. Schreierb, G. Brezesinskic, H. Fabiand, H. W. Meyere and P. Nuhna, J. Controlled Release, 1994, 30, 105-116.

15 K. I. Izutsu, C. Yomota and T. Kawanishi, J. Pharm. Sci., 2011, 100, 2935-2944.

16 J. H. Crowe, L. M. Crowe, J. F. Carpenter, A. S. Rudolph, C. A. Wistrom, B. J. Spargo and T. J. Anchordoguy, Biochim. Biophys. Acta, 1988, 947, 367-384.

17 T. D. Madden, M. B. Bally, M. J. Hope, P. R. Cullis, H. P. Schieren and A. S. Janoff, Biochim. Biophys. Acta, 1985, 817, 67-74.

18 D. C. Cipolla, H. Wu, S. Eastman, T. Redelmeier, I. Gonda and H.-K. Chan, J. Pharm. Sci., 2014, 103, 314-327, DOI: 10.1002/jps.23795.

19 H. X. Ong, D. Traini, D. Cipolla, I. Gonda, M. Bebawy, H. Agus and P. Y. Young, Pharm. Res., 2012, 29, 3335-3346.

20 M. Almgren, E. Edwards and G. Karlsson, Colloids Surf., A, 2000, 174, 3-21.

21 O. A. Glatter, J. Appl. Crystallogr., 1977, 10, 415-421.

22 B. Stark, G. Pabst and R. Prassl, Eur. J. Pharm. Sci., 2010, 41, 546-555.

23 L. F. Siow, T. Rades and M. H. Lim, Cryobiology, 2007, 55, 210-221.

24 L. F. Siow, T. Rades and M. H. Lim, Cryobiology, 2008, 57, 276-285.

25 H. Talsma, M. J. van Steenbergen and D. J. Crommelin, Cryobiology, 1992, 29, 80-86. 
26 M. S. Webb, N. L. Boman, D. J. Wiseman, D. Saxon, K. Sutton, K. F. Wong, P. Logan and M. J. Hope, Antimicrob. Agents Chemother., 1998, 42, 45-52.

27 C. A. Schneider, W. S. Rasband and K. W. Eliceiri, Nat. Methods, 2012, 9, 671-675.

28 C. Bonnaud, C. A. Monnier, D. Demurtas, C. Jud, D. Vanhecke, X. Montet, R. Hovius, M. Lattuada, B. Rothen-Rutishauser and A. Petri-Fink, ACS Nano, 2014, 8, 3451-3460.

29 B. Michen, C. Geers, D. Vanhecke, C. Endes, B. RothenRutishauser, S. Balog and A. Petri-Fink, Sci. Rep., 2015, 5(9793), 1-7.

30 D. N. Mastronarde, J. Struct. Biol., 2005, 152, 36-51.

31 N. M. Kirby, S. T. Mudie, A. M. Hawley, D. J. Cookson, H. D. T. Mertens, N. Cowieson and V. A. Samardzic-Boban, J. Appl. Crystallogr., 2013, 46, 1670-1680.
32 J. Brunner-Popela and O. Glatter, J. Appl. Crystallogr., 1997, 30, 431-442.

33 B. Weyerich, J. Brunner-Popela and O. Glatter, J. Appl. Crystallogr., 1999, 32, 197-209.

34 G. Fritz and O. Glatter, J. Phys.: Condens. Matter, 2006, 18, S2403-S2419.

35 O. Glatter, J. Appl. Crystallogr., 1979, 12, 166-175.

36 O. Glatter, J. Appl. Crystallogr., 1981, 14, 101-108.

37 D. E. Koppel, J. Chem. Phys., 1972, 57, 4814-4820.

38 FDA Guidance for Industry: Modified Release Solid Oral Dosage Forms: Scale-Up and Post Approval Changes (SUPAC-MR): Chemistry, Manufacturing and Controls; In Vitro Dissolution Testing and In Vivo Bioequivalence Documentation, September, 1997. 